\title{
Defects of Bénard cell on a propagating front
}

Cite as: Phys. Fluids 32, 024107 (2020); https://doi.org/10.1063/1.5126355

Submitted: 02 September 2019 . Accepted: 06 November 2019 . Published Online: 10 February 2020

Di Wu (吴笛) (D), Li Duan (段俐) (D), and Qi Kang (康琦) (D)

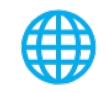

\section{ARTICLES YOU MAY BE INTERESTED IN}

Anisotropic particles in two-dimensional convective turbulence

Physics of Fluids 32, 023305 (2020); https://doi.org/10.1063/1.5141798

Numerical investigations on the flow over cuboids with different aspect ratios and the emitted noise

Physics of Fluids 32, 025103 (2020); https://doi.org/10.1063/1.5131827

Hydrodynamic behavior of two-dimensional tandem-arranged flapping flexible foils in uniform flow

Physics of Fluids 32, 021903 (2020); https://doi.org/10.1063/1.5133794

\section{Sign up for topic alerts} New articles delivered to your inbox 


\title{
Defects of Bénard cell on a propagating front
}

\author{
Cite as: Phys. Fluids 32, 024107 (2020); doi: 10.1063/1.5126355 \\ Submitted: 2 September 2019 • Accepted: 6 November 2019 • \\ Published Online: 10 February 2020
}

Di Wu (吴笛), 미 Li Duan (段俐), ${ }^{1,2, a)}$ (D) and Qi Kang (康琦) ${ }^{1,2, a)}$ (D)

\begin{abstract}
AFFILIATIONS
${ }^{7}$ Key Laboratory of Microgravity (National Microgravity Laboratory), Institute of Mechanics, Chinese Academy of Sciences, Beijing 100190, China

${ }^{2}$ School of Engineering Science, University of Chinese Academy of Sciences, Beijing 100049, China
\end{abstract}

a) Authors to whom correspondence should be addressed: duanli@imech.ac.cn and kq@imech.ac.cn

\begin{abstract}
Bénard-Marangoni convection can be used to self-organize hexagonal convective cells, but defects easily emerge in the hexagonal pattern, which hinders its application in industry. The dynamics of front propagation and defect generation are studied in this paper. We focus especially on the onset process of a local disturbance of a hexagonal pattern, named the "nucleus." The front propagation of the nucleus has been researched through numerical simulations of a model equation and experiments. In the numerical simulations, a single nucleus can evolve into a perfect hexagon pattern under critical or subcritical conditions, and a random disturbance can generate multiple nuclei which evolve into grain boundaries. In addition, under supercritical conditions, defects also emerge as a single nucleus grows. The instability of front propagation is considered to be the mechanism for the generation of irregular patterns. The curvature effect makes the protrusion of the front have a larger velocity in supercriticality, which results in a wavy front, and defects are generated in the concave portion of the front. Also, because of the curvature effect, the front of an irregular pattern has a larger velocity than that of the regular pattern since the protrusion of the front in the irregular pattern increases the average velocity. Experiments have also been carried out by using an infrared camera to analyze front propagation. The results are qualitatively in agreement with the results of numerical simulations. Through the study of defect generation in front propagation, we put forward a method for generating a hexagon pattern which greatly reduces the number of defects.
\end{abstract}

Published under license by AIP Publishing. https://doi.org/10.1063/1.5126355

\section{INTRODUCTION}

Bénard convection in a thin liquid layer, caused by thermocapillary effect (Marangoni effect), forms cellular convection. As early as 1900, Bénard ${ }^{1}$ observed hexagonal cells when heating a shallow liquid layer. Lord Rayleigh ${ }^{2}$ gave the critical $R a$ number through linear stability analysis and attributed this phenomenon to buoyant instability. However, Pearson ${ }^{3}$ revealed the truth that thermocapillary instability is the mechanism of Bénard convection in a thin layer. The self-organization of hexagonal Bénard cells attracted extensive attention in pattern formation. Cloot and Lebon ${ }^{4}$ explained the mechanism of hexagonal pattern formation through weakly nonlinear analysis. Hexagonal cells are generated by the resonant interaction of triads of waves oriented at $120^{\circ} .5$ Theoretical studies, including linear stability analysis and weakly nonlinear analysis, are based on the Navier-Stokes equation and need to carry out complex calculations and derivations. Therefore, Swift and Hohenberg ${ }^{6}$ proposed a simplified model equation to describe the
Bénard pattern. This simplified equation qualitatively explains the self-organization of both the hexagon pattern and stripe pattern. Bestehorn ${ }^{7}$ proposed a more accurate model equation by considering the nonlinear interaction between the critical wave $\left(k_{\mathrm{c}}\right)$ and the harmonic $\left(2 k_{\mathrm{c}}\right)$.

In recent years, some new applications of Bénard convection have been explored. By utilizing the self-organization of Bénard cells, functional materials with regular patterns, such as the hexagonal porous film, gold nanocrystal, and array of lenses, have been produced. ${ }^{8-10}$ However, defects in self-organization are uncontrollable, which is a challenge for the industrial application of Bénard cells. Koschmieder and Switzer ${ }^{11}$ conducted a large number of experiments to study critical convection. Defects in different structures, such as heptagon, pentagon, quadrilateral, triangular, and even starlike structures, were found in their experiments. They conjectured that the "star" defect was generated by the inhomogeneity of the experimental apparatus. However, Kvarving et al. ${ }^{12}$ thought that the "star" defect could also appear even if the uniformity of the 
boundary is perfect because they observed the pattern in numerical simulations. Cerisier et al. ${ }^{13,14}$ researched the defects of Bénard cells through methods of statistics and topology. They described typical defect as a pentagon-heptagon pair and a "flower" pattern. It was found that the hexagonal or cylindrical vessel minimized the disorder. Schatz and Neitzel ${ }^{15}$ concluded the order-disorder transition as a secondary instability of $\operatorname{Pr} \sim 1000$ when they reviewed Cerisier et al.'s work. ${ }^{13,14}$

The formation of disorder is caused, in a large extent, by the boundary effect and initialization. In the Rayleigh-Bénard convection, the boundaries tend to orient convective rolls perpendicular to them. This phenomenon can be found in the experiment conducted by Morris et al. ${ }^{16}$ They used a circular boundary which resulted in many defects, i.e., dislocation, disclination, focus singularity, and dislocation. Recently, Tripathi and Kumar ${ }^{17}$ studied the effect of initial condition and boundary on defect evolution by simulating the Swift-Hohenberg equation. In Marangoni-Bénard convection, the boundaries prefer cells with edges perpendicular to it. Therefore, it is easier to form regular hexagonal cells in a hexagonal or equilateral triangular vessel than in a rectangular vessel. Most experiments (Bénard, ${ }^{1}$ Koschmieder, ${ }^{11}$ and Schat ${ }^{18}$ ) were carried out in a triangular or circular vessel with good uniformity. However, they did not control the initial condition. Cerisier et al. ${ }^{13,14}$ used a thermal marking technique to impose regular arrays of triangles, squares, and hexagons. This studied the disordered formation by extrinsic effect after the initialization of regular patterns. For the purpose of studying the extrinsic effect, they chose a hexagon vessel to reduce the boundary effect. They found that the hexagon pattern in the hexagonal vessel is stable if the wavelength is equal to the natural one, and otherwise, the pattern evolves to defects.

There is a special case of destabilization: hexagon cells first emerge in the local region and then spread to the whole region. The theoretical study of front propagation was conducted by analyzing the amplitude equation. Pulled fronts under supercritical conditions and pushed fronts under subcritical conditions were discussed. $^{19-21}$ Tzella and Vanneste ${ }^{22}$ simulated the chemical front in a cellular flow. Tripathi and Kumar ${ }^{17}$ studied the $1 \mathrm{D}$ front propagation of periodic and chaotic patterns using the SwiftHohenberg equation and Ginzberg-Landau equation, respectively. Fineberg ${ }^{24}$ conducted an experiment to study the front propagation of Rayleigh-Bénard convection. To the best of our knowledge, no research has been carried out on the effect of front propagation on pattern formation in Bénard-Marangoni convection. Can defects emerge on the fronts of regular hexagonal patterns? How do the fronts induce the generation of defects? How can we restrain disorder patterns? All these questions require further research.

Combining numerical simulations of the model equation and experiments on Bénard-Marangoni convection, this paper aims to explore the dynamics of front propagation and its effect on pattern formation. Section II introduces the model equation, numerical simulation method, and experimental method. Section III presents the numerical results of front propagation and defect generation and makes qualitative comparisons between the simulation and experiment. We give an explanation for the defects generated in front propagation and put forward a method for generating the regular pattern. A conclusion is given in Sec. IV.

\section{NUMERICAL AND EXPERIMENTAL METHODS}

\section{A. Model equation and numerical method}

Bénard convection is described accurately by the Navier-Stokes equation with the Marangoni boundary condition. However, the complexity of the Navier-Stokes equation brings great difficulty to research the dynamics of 2-dimensional patterns. Therefore, many simplified equations have been proposed to describe pattern formation. The most famous one is the Swift-Hohenberg equation, which is normalized as

$$
\frac{\partial \phi}{\partial t}=\varepsilon \phi-(\Delta+1)^{2} \phi+\delta \phi^{2}-\phi^{3},
$$

where $\varepsilon$ is the supercritical number. If the supercritical number $\varepsilon \geq 0$, the solution $\phi=0$ is unstable, resulting in pattern formation. $\delta \neq 0$ is set to simulate Marangoni-Bénard convection in which hexagonal patterns are obtained. $\delta=0$ is set to simulate Rayleigh-Bénard convection in which striped patterns are obtained.

Bestehorn ${ }^{7}$ had put forward a modified equation with a higherorder approximation to the Navier-Stokes equation,

$$
\begin{aligned}
\dot{\psi}= & {\left[\gamma_{1} \varepsilon-\gamma_{2}\left(k_{c}^{2}+\Delta_{2}\right)^{2}\right] \psi(x, t)-a_{1}\left[\nabla_{2} \psi(x, t)\right]^{2} } \\
& -a_{2} \psi(x, t) \Delta_{2} \psi(x, t)-b_{1} \psi(x, t)\left[\Delta_{2} \psi(x, t)\right]^{2} \\
& -b_{2} \psi(x, t) \Delta_{2}^{2} \psi^{2}(x, t) .
\end{aligned}
$$

Similar to the Swift-Hohenberg equation, the linear terms in Eq. (2) describe an exponential growth of the disturbance wave. The critical wave number is $k_{c}$. However, the nonlinear terms in Eq. (2) give a more precise description of the nonlinear interaction between waves. The parameters $a_{1}, a_{2}, b_{1}$, and $b_{2}$ are determined by nonlinear analyses of the critical wave $\left(k_{\mathrm{c}}\right)$ and the harmonic $\left(2 k_{\mathrm{c}}\right)$. The parameters for a pure Rayleigh-Bénard flow and pure Marangoni-Bénard flow are given in Table $\mathrm{I}$.

In order to simulate the physical boundary, we adopt the following solid-wall boundary conditions:

$$
\partial_{\mathrm{n}} \psi(x, t)=\psi(x, t)=0 .
$$

When carrying out numerical simulation, the coefficients on the right of Eq. (2) are summarized into a linear operator L and a nonlinear operator $\mathrm{N}$,

$$
\frac{\partial \psi}{\partial t}=L(\psi)+N(\psi)
$$

Therefore, Eq. (2) can be divided into homogeneous equations and a nonhomogeneous equation,

$$
\text { Nonhomogeneous equation: } \frac{\partial \psi^{\text {in }}}{\partial t}=L\left(\psi^{\text {in }}\right)+N\left(\psi^{\text {in }}\right) \text {, }
$$

TABLE I. The parameters for Rayleigh-Bénard flow and Marangoni-Bénard flow.

\begin{tabular}{lccccccc}
\hline \hline & $k_{c}$ & $\gamma_{1}$ & $\gamma_{2}$ & $a_{1}$ & $a_{2}$ & $b_{1}$ & $b_{2}$ \\
\hline RB flow & 2.17 & 7.5 & 0.085 & 2.2 & 1.1 & -0.01 & 0.19 \\
MB flow & 2.03 & 6.2 & 0.073 & 4.6 & 0.2 & -0.29 & 0.31 \\
\hline \hline
\end{tabular}




$$
\text { Homogeneous equations: }\left\{\begin{array}{c}
L\left(\psi^{\mathrm{ha}}\right)=0, \\
\psi^{\mathrm{ha}}=-\psi^{\text {in }}, \\
\partial_{n} \psi^{\mathrm{ha}}=-\partial_{n} \psi^{\text {in }} .
\end{array}\right.
$$

The nonhomogeneous equation is solved by the Fourier spectral method. It provides the boundary condition for the homogeneous equations in each time step. The homogeneous equations are solved by the Gauss-Seidel iteration. The size of the calculation is $240 \times 240$, and the discrete grid is $240 \times 240$. The time derivative uses a second-order frog leap implicit format. The solution of Eq. (2) is given by $\psi=\psi^{\text {in }}+\psi^{\text {ha }}$.

To study the initial condition effect on pattern formation, two different initial conditions are taken into account:

(1) Global random disturbance [Fig. 1(a)], where the initial disturbances are set random values distributed in the range of $[0,0.01]$ to the nodes.

(2) Local hexagonal disturbance [Fig. 1(b)], where it is assumed that there is a local hexagonal pattern in the center, which evolves to a global pattern as the fronts of disturbance propagate.

When the physical system is linearly unstable, any kind of small disturbance will grow exponentially and eventually create various patterns. The natural disturbance is uncertain and stochastic, so the global random disturbance is more similar to the naturally formed Bénard cells. However, the local hexagonal disturbance appears in some special cases, which are rarely studied. We focus on the second case and aim to study the dynamic process of front propagation and the formation of disorder patterns during the process. The hexagonal pattern is obtained by superimposing triads of waves oriented at $120^{\circ}$,

$$
\psi=A(\vec{r})\left[\cos \left(k_{1} \vec{r}\right)+\cos \left(k_{2} \vec{r}\right)+\cos \left(k_{3} \vec{r}\right)\right] / 3,
$$

where wave vectors $k_{1}, k_{2}$, and $k_{3}$ are oriented at $120^{\circ}$. The amplitude of waves $A(\vec{r})$ is assumed as a Gaussian function,

$$
A(\vec{r})=A_{0} \exp \left(-\vec{r}^{2} / \sigma^{2}\right),
$$

where $A_{0}$ is the maximum amplitude and $\sigma$ is set to be $\pi / k_{c}$, which is a half of the cell size.

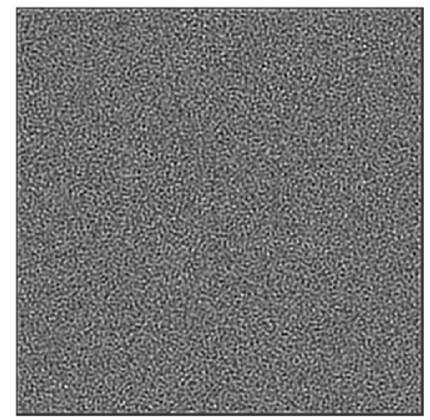

(a)

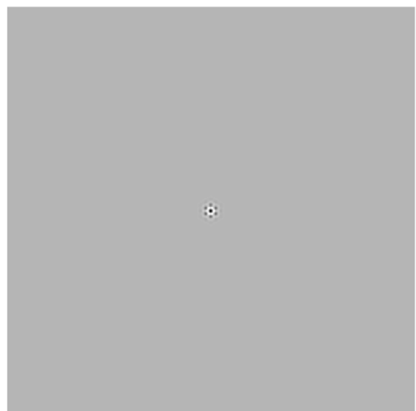

(b)
FIG. 1. Initial conditions at $t=0$ : (a) global random disturbance and (b) local hexagonal disturbance. Local hexagons can be found at the center of Fig. 1(b).

\section{B. Experimental method}

We carried out experiments in an open rectangular cavity with a size of $160 \mathrm{~mm} \times 160 \mathrm{~mm}$. KF96-50 cSt silicone oil made by Shin-Etsu is selected as the fluid medium. A thin layer with depth $d<3 \mathrm{~mm}$ is studied to diminish the buoyant effect. The layer is heated from the bottom of the cavity by an embedded heating film. The bottom plate is a sandwich structure of copper, silica gel, and aluminum to guarantee high thermal uniformity. Since BénardMarangoni convection is caused by the surface temperature gradient, the surface temperature captured by an infrared camera can well reflect the flow pattern. To visualize convective patterns, an infrared camera is adopted to capture the distribution of surface temperature. More details of the experimental setup can be found in Ref. 25. When the temperature difference reaches the critical value, the stationary fluid starts to form a convective pattern. We use the supercritical number $\varepsilon$ to describe the state of convection. As the temperature difference is not directly measured, we define the supercritical number $\varepsilon$ as

$$
\varepsilon=\frac{T_{\mathrm{b}}-T_{\mathrm{bc}}}{T_{\mathrm{bc}}-T_{\mathrm{e}}}
$$

in which $T_{\mathrm{b}}$ is the bottom temperature, $T_{\mathrm{e}}$ is the environmental temperature, and $T_{\mathrm{bc}}$ is the bottom temperature when cells begin to appear.

Critical convection usually forms the global pattern, and the local pattern and front propagation are only observed in a few cases. To generate the local pattern, we placed four cover plates at $30 \mathrm{~mm}$ above the bottom, and the gaps of the plates form a window, as shown in Fig. 2. The main purpose for using cover plates is to restrain air convection and reduce heat dissipation to the interface, which directly reduces the temperature difference of the liquid layer. Therefore, the open area under the window and the area covered by the plates are distinct in temperature difference under a thermal equilibrium state. In the open area, the heat transfer of the liquid surface is induced mainly by natural convection with a high heat dissipation rate, so the surface temperature is lower, and the temperature difference is larger. However, in the area under the cover plates, natural convection is restrained with poor heat transfer, so the surface temperature is high and the temperature difference is small. Therefore, local hexagon cells form near the window, where

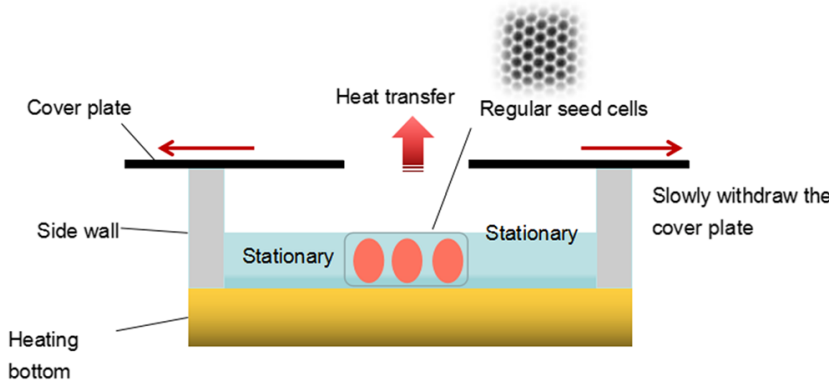

FIG. 2. The schematic of the new method for the generation of hexagonal Benard cells. There are four cover plates, but only two cover plates are showed here because this figure is a two-dimensional side-view sketch. 
the temperature difference is higher than the critical value. We initialized the local pattern first by using the cover plates and then by removing them to study front propagation, as shown in Sec. III B. We also slowly withdraw the cover plates to make a regular hexagon pattern, as shown in Sec. III D.

The accuracy of the experiment is affected by the temperature accuracy, the flatness and the levelness of the bottom surface. The temperature is controlled with a small fluctuation of $\pm 0.01^{\circ} \mathrm{C}$ and an accuracy of $\pm 0.2^{\circ} \mathrm{C}$. The thermal uniformity of the copper bottom plate is $0.02{ }^{\circ} \mathrm{C}$, and the flatness is $0.01 \mathrm{~mm}$. We carefully adjusted the levelness to less than $0.005^{\circ}$, which causes a thickness difference of $0.014 \mathrm{~mm}$. Our experiment requires good homogeneity of the physical system in the horizontal directions. Here, we estimate the influence of bottom fluctuation or the inclination to the uniformity of the supercritical number. According to the critical temperature, which is $4{ }^{\circ} \mathrm{C}$ for a $2 \mathrm{~mm}$ layer with $50 \mathrm{cSt}$ silicone, ${ }^{25}$ the uniformity of the supercritical number is $2 \%$ in the horizontal directions.

\section{RESULTS AND DISCUSSION}

\section{A. Instability near the onset}

In a liquid layer with a vertical temperature gradient, Bénard convection starts when the temperature gradient is beyond a certain threshold. The corresponding threshold in the model equation is $\varepsilon=0$, which means that the pattern occurs when $\varepsilon>0$. The critical pattern near the onset was expected to form a perfect regular pattern, but in fact the regularity of the pattern, to a large extent, depends on the initial condition. Therefore, we want to find a proper initialization pattern which can automatically develop a regular pattern. We are interested in the pattern generated by the expansion of local disturbance. If the local disturbance is the nucleus of a regular hexagon, the final pattern is supposed to be a regular hexagon.

Figure 3 shows the front propagation process after the initialization of local hexagonal disturbance [Fig. 1(b)] with $\varepsilon=0.001$. The local hexagon is considered as a single nucleus, and it develops into a hexagon cell with a circular front when $t=200$. In mathematics, the front is a special solution which is the transition from one solution to another solution in space. Here, the hexagonal pattern is a stable solution and the uniform state is an unstable solution near the critical. The front solution means that one side is a hexagonal pattern and the other side is a uniform state. The front is the region between the two red dashes, as shown in Fig. 3(a). On the front, the amplitude of the hexagonal pattern gradually decreases to 0 , so there is a blurred or weak hexagon near the edges. As shown in Figs. 3(a)-3(d), the front propagates steadily and the newly generated cells are very regular and without disorder. Although the rectangular vessel may easily cause defect due to the boundary effect, this simulation shows that the hexagon cells keep the regular arrangement even when they occupy the whole region. This means that the intrinsic property of the hexagon pattern dominates the boundary effect. During front propagation, new generations of the pattern maintain the original pattern's orientation. This gives us an inspiration to develop a method to reduce defects by exerting a single nucleus of hexagon.

The instability of random initialization is studied for comparison. Figures $4(\mathrm{a})-4$ (d) show the pattern evolution from random

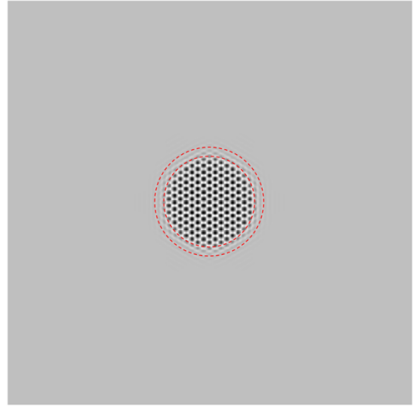

(a)

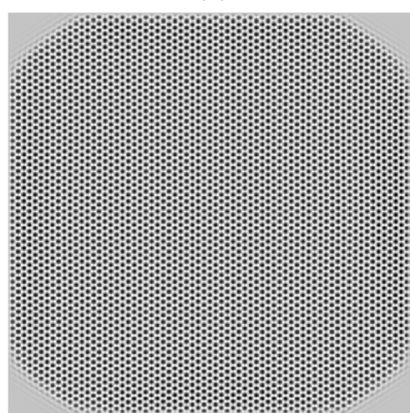

(c)

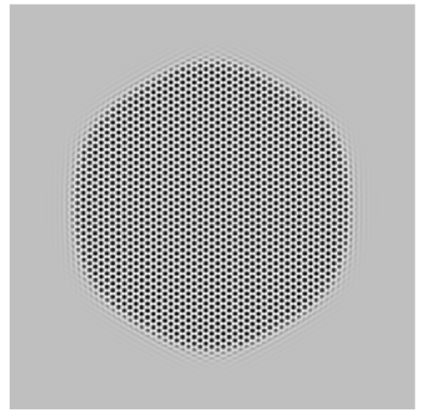

(b)

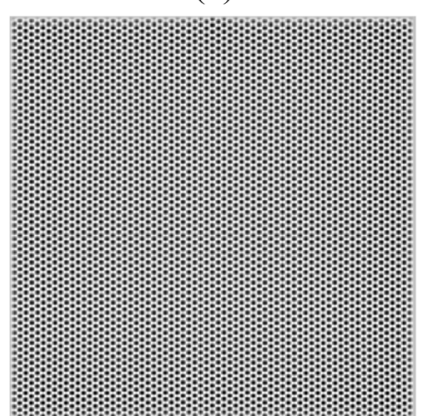

(d)
FIG. 3. The evolution of local hexagonal disturbance: (a) pattern at $t=200$, (b) pattern at $t=400$, (c) pattern at $t=600$, and (d) pattern at $t=800$. The front is marked by red dashed lines in Fig. 3(a).

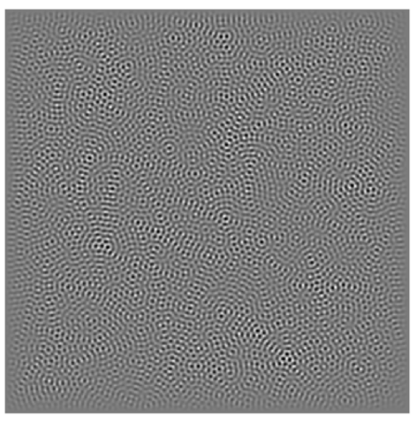

(a)

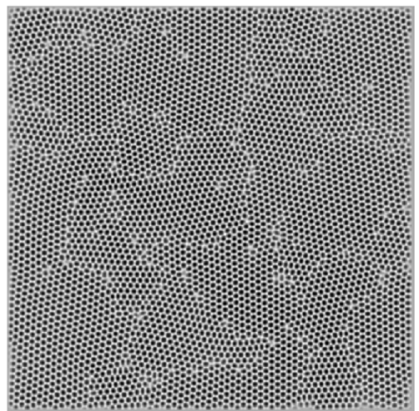

(c)

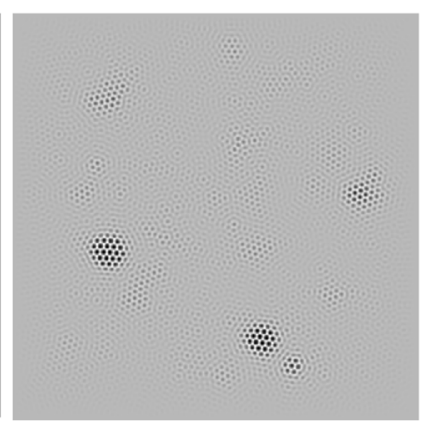

(b)

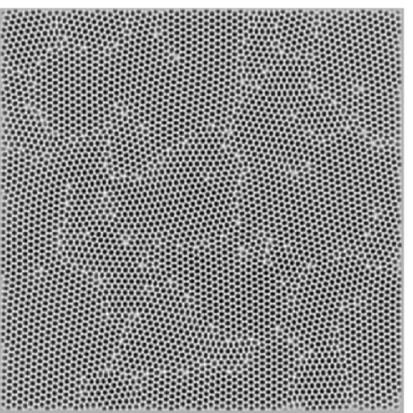

(d)
FIG. 4. The evolution of global random disturbance: (a) pattern at $t=200$, (b) pattern at $t=400$, (c) pattern at $t=600$, and (d) pattern at $t=800$. 
initialization [shown in Fig. 1(a)]. It is found that the final pattern contains many defects, and the defects cluster to form grain boundaries. As shown in Fig. 4(c), grain boundaries divide the hexagonal patterns into many domains. Each domain consists of a regular pattern whose orientation is different from those of the adjacent domains. Grain boundary formation can be explained by the growth of multiple nuclei. As shown in Fig. 4, the amplitude of random disturbance grows uniformly at the beginning [Fig. 4(a) $t=200$ ], and then the amplitude accumulates in some scattered locations and the strong multiple nuclei of the hexagonal pattern [Fig. 4(b) $t=400$ ]. The accumulation of amplitude is conjectured as the nonlinear action of the weak pattern. These scattered nuclei with different oriented hexagons grow and form grain boundaries. The grain boundaries are a very stable structure, and no obvious change is found between Figs. 4(c) and 4(d).

Although the front propagation of a single nucleus can form a regular pattern, this is hardly found in naturally formed patterns in experiments. Figures 5(a)-5(d) show a local hexagonal pattern that automatically formed in a $50 \mathrm{cSt}$ silicone layer of $2.30 \mathrm{~mm}$ depth. The characteristic time for thermal diffusion is defined as $d^{2} / \kappa$, which is 49.4 s. So the nondimensional time $\tilde{T}$ is defined as $t /\left(d^{2} / \kappa\right)$. Cells form in the central region and then the front propagates outward until the cells occupy the whole region. This experiment demonstrates that a propagating front can naturally occur near the onset. The final pattern contains defects because the initial pattern is irregular. However, the newly generated pattern has fewer defects than the initial pattern, which means that it is possible to obtain a regular pattern by front propagation.

Front propagation is circular in the simulation while it is not circular in the experiment. This is because the simulation is an idealized model. In the numerical simulation, we simplify the

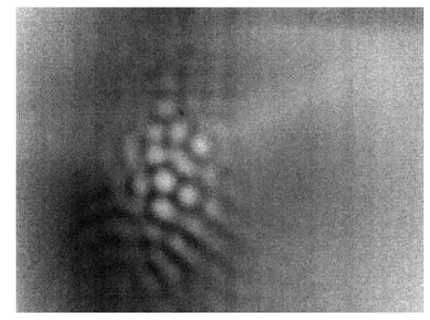

(a)

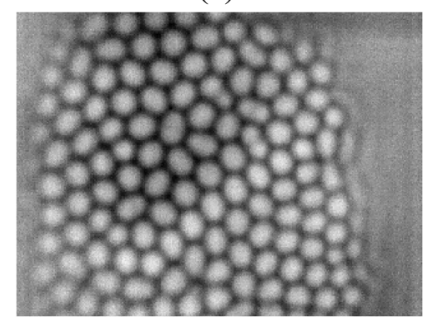

(c)

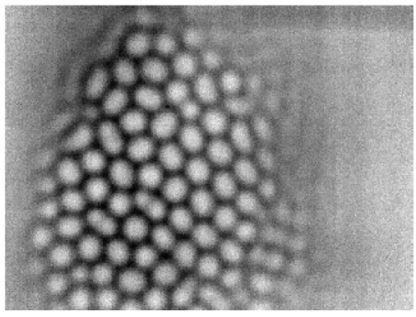

(b)

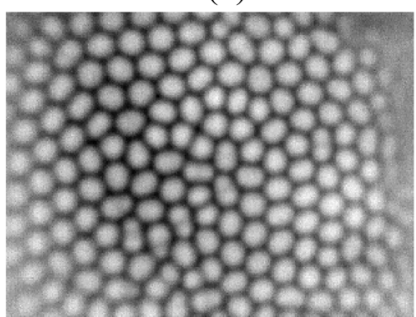

(d)
FIG. 5. The onset of Marangoni-Bénard convection (50 cSt, $d=2.30 \mathrm{~mm})$ : (a) pattern at $t=0 \mathrm{~s}(\tilde{T}=0)$, (b) pattern at $t=300 \mathrm{~s}(\tilde{T}=6.07)$, (c) pattern at $t=600$ $(\tilde{T}=12.15)$, and (d) pattern at $t=900(\tilde{T}=18.22) . \tilde{T}$ is the nondimensional time, defined as $t /\left(d^{2} / \kappa\right)$. initial disturbance into two representative cases: local hexagonal disturbance and global random disturbance. The real situation is a very complex case, which is the combination of these two situations or the emergence of multiple nuclei. Comparing Figs. 3 and 5, a circular pattern was initialized in the simulation but the initial pattern in the experiment is not circular. Moreover, to observe a circular front in experiments, good homogeneity is required. However, the homogeneity of our open cavity is hardly ensured because the air convection above the interface may disturb the front propagation. It also requires high uniformity of temperature distribution, a good flatness, and a small inclination on the bottom plate, as well as uniform interface heat dissipation. These limitations make it difficult to observe circular front propagation in experiments.

\section{B. The velocity of front propagation}

To study front velocity, it is necessary to give a criterion which determines the position of the front. As the pattern intensity decreases continuously to zeros on the front, we define the front as the point where the amplitude is equal to $20 \%$ of that of the regular pattern. First, we need to calculate pattern amplitude distribution, by taking the weighted RMS of the disturbance in the adjacent area. Then, we calculate the area of the pattern region where the amplitude is larger than $20 \%$ of the regular pattern's amplitude. Finally, the average radius of the front is calculated from the area of the pattern region. In numerical simulation, when the front of the cellular region propagates outward, it is circular. So, the radius is calculated by $\sqrt{S / 2 \pi}$, where $S$ is the area of the cellular region.

Figure 6(a) shows the time evolution of the front radius at a different supercritical number $\varepsilon$. The slope of the curve represents the velocity of the front. Interestingly, the velocity of the front is not a constant value and it is classified into 3 stages: (1) curvature flow, (2) regular pattern, and (3) defect pattern.

(1) Curvature flow. As the name suggests, the velocity of the front is related to the front curvature. The curvature effect is prominent in the early stage because the front of a small circle has a large curvature. Taking a cross section of the nucleus, we can see that the left front and the right front are very close to each other, so the interaction between the fronts is prominent. In the supercritical case, the "force" between the fronts is repulsive, so the velocity is greater at the beginning. In the subcritical case, the force between the fronts is attractive, so the velocity is smaller at the beginning. As the curvature effect shrinks the hexagonal region under the subcritical condition, a positive velocity for $\varepsilon=-0.005$, as shown in Fig. 6(a), is obtained by initializing a large region. We simulate the front propagation under $\varepsilon=0.005$ and wait until the diameter is equal to about 18 and then simulate the front propagation under $\varepsilon=-0.005$. To distinguish the different initializations, we define $t=100 \mathrm{~s}$ in Fig. 6(a) as the initial time for $\varepsilon=-0.005$.

(2) Regular pattern. As the radius increases, the effect of the curvature can be neglected. Due to the marginal or subcritical instability, the newly generated cells maintain a regular hexagon pattern during the whole process. A straight line is found, as shown in Fig. 6(a), when $\varepsilon=0.01$, which is close to 


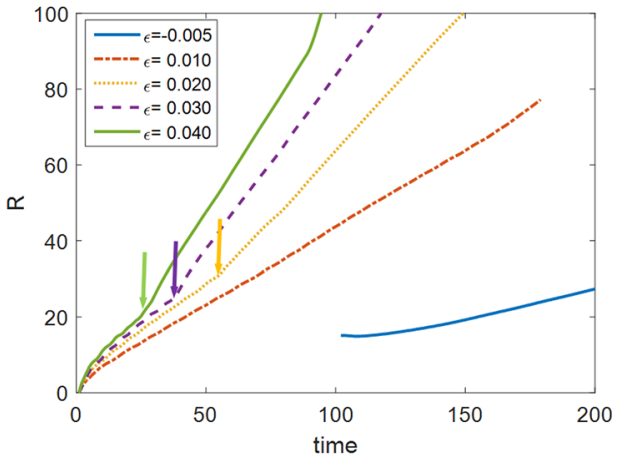

(a)

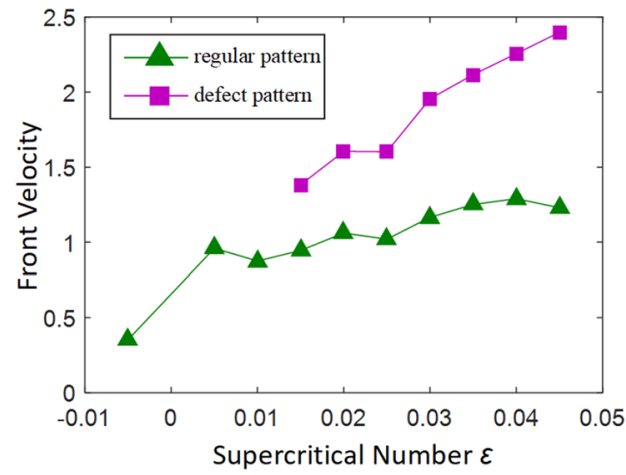

(b) the critical condition with the velocity of front propagation being constant. In the supercritical case, the regular hexagon pattern transits to defects as the front propagates.

(3) Defect pattern. The velocity of front propagation drastically increases when the pattern becomes irregular. For instance, the curve shown in Fig. 6(a) with $\varepsilon=0.02$ has two slopes, and they represent the change of front velocities. The transition point of velocity of front propagation just corresponds to the pattern transformation from a regular pattern to an irregular pattern.

Figure 6(b) gives the velocities of the regular pattern and defect pattern under a different supercritical number. By selecting a different linear segment, as shown in Fig. 6(a), the velocities of the regular pattern and defect pattern are obtained from linear fitting. The curvature effect is excluded. According to the theory of front propagation, the velocity of front propagation depends on the supercritical number $\varepsilon$. When the supercritical number $\varepsilon \gg 0$, the front is a pulled front, whose velocity is determined by the front profile. The velocity of the pulled front is proportionate to $\sqrt{\varepsilon}$. However, the front of the defect pattern propagates 1.5-2 times faster than that of the regular pattern. This phenomenon has not been predicted or explained by the present theory.
It is found that the velocity of front propagation does not decrease to zero even under the subcritical condition, $\varepsilon=-0.005$, which means the pattern expands when the temperature difference is lower than the critical value. In this case, the front is known as a pushed front because both the uniform state and hexagon state are stable and the velocity of front propagation is determined by the potential difference between the two states. The velocity of front propagation is obviously affected by the curvature effect, which makes the pattern region shrink. As a result, the front velocity presents a negative value when the diameter of the pattern region is small; if the local disturbance radius is large enough to exclude the curvature effect, the velocity of front propagation will be a positive value. This also explains well the hysteresis phenomenon: the pattern region with a large diameter will not disappear under a slight subcritical condition.

In this experiment, we studied the front propagation in one dimension for simplicity. One-dimensional front propagation is accomplished by installing two cover plates above the liquid layer, which suppress the pattern beneath the plate due to poor heat transfer. The gap between the two cover plates is about $70 \mathrm{~mm}$, which allows the front of the pattern to propagate through the gap. The thickness of the layer is $2.00 \mathrm{~mm}$, and hexagonal cells are initialized on one side of the gap. Figure 7 presents the experimental

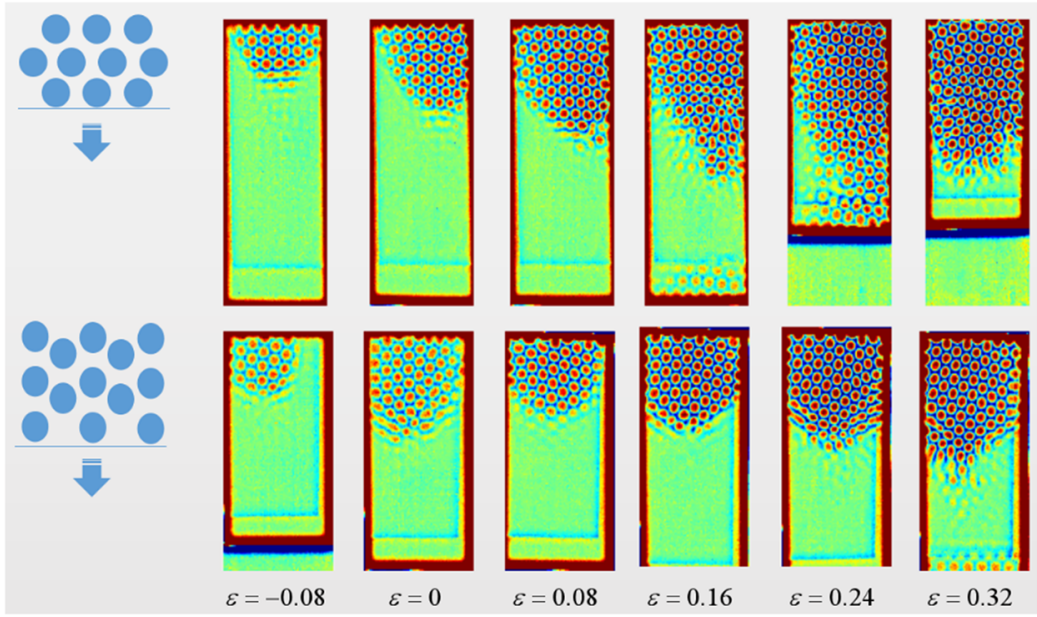

FIG. 7. The front propagation of Bénard cells in one dimension. 
results of Bénard cells under different supercritical numbers when $t=66 \mathrm{~s}$. To take into account the effect of cellular orientation to the front velocities, we studied hexagons in two orientations, with the cell array parallel or vertical to the front. At the same propagating time, the area of the cellular region gets larger as the supercritical number increases. This implies that the velocity of front propagation increases with the increase in the supercritical number. Also, the experiments indicate that the velocity of front propagation with the parallel cells is larger than that of the vertical cells.

The direct relation between front acceleration and the defect pattern is found. The objective of Fig. 8 is to show the relation between the irregular patterns the acceleration of front propagation. The left picture of Fig. 8 shows the average position of the front vs time. The average position of the front is calculated through the pattern area according to the front criterion. The right picture in Fig. 8 is the final pattern. The $\mathrm{Y}$ axes of both pictures are consistent. As the front propagates from the top to the bottom, we find that the regular hexagonal pattern develops into a defect pattern which consists of quadrilateral, pentagonal, hexagonal, and heptagonal cells. The red line is the dividing line between the regular pattern and the irregular pattern in the right figure. Interestingly, the red line corresponds to the transition point of velocity in the left figure. By analyzing the two figures combined, we can clearly observe that the defects appear when the front is accelerating. This phenomenon is found in most experiments, which validates the prediction of simulation.

Figure 9 gives the front velocities of the vertical cellular array and parallel cellular array under different supercritical numbers. The velocity is nondimensionalized by the characteristic length $\mathrm{d}$ and the characteristic time $d^{2} / \kappa$. The nondimensional velocity is $\tilde{V}=(\kappa / d) v=19.81 v$ for a $50 \mathrm{cSt}$ silicone layer of $2.00 \mathrm{~mm}$ depth. The experimental result of velocity is 5 times higher than that from the model equation when $\varepsilon \approx 0$. It is impractical to obtain a precise agreement between the weakly nonlinear model and the experiment, but some qualitative agreements can be found. The velocity of front propagation increases with the increase in the supercritical number, and the pulled front is proportional to $\sqrt{\varepsilon}$. Under the subcritical condition, the experiments show subcritical bifurcation when $-17 \%<\varepsilon<0 \%$, and the velocity of the pushed front is larger than 0 . These results are qualitatively consistent with the model equation.

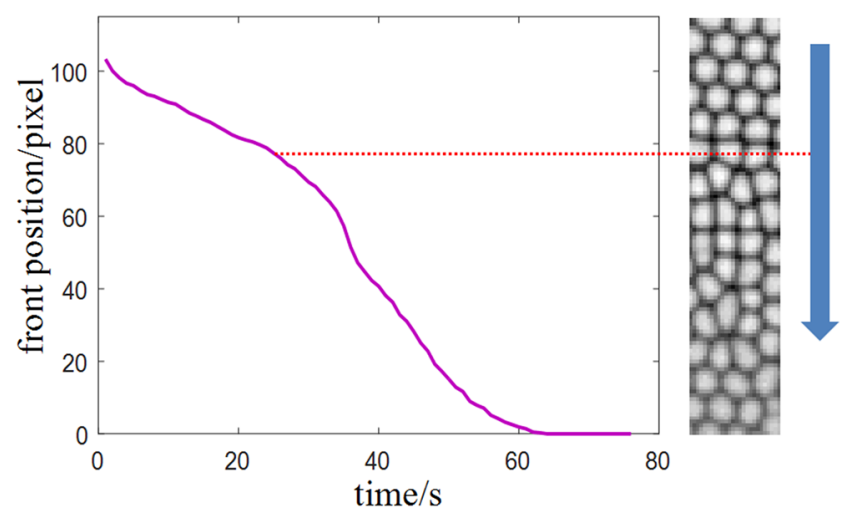

FIG. 8. The evolution of the front position and the final pattern.

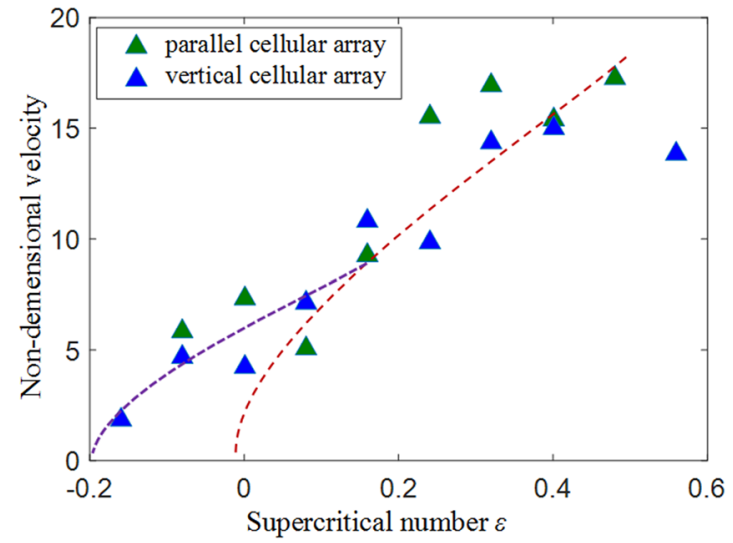

FIG. 9. The nondimensional velocity of the front.

\section{The mechanism of defect pattern}

When a front is propagating, whether the newly generated pattern is regular or irregular is related to the velocity of front propagation. The formation of an irregular pattern in a propagating front is caused by the instability of front propagation, which forms a wavy front. The instability of a front is determined by the curvature effect of velocity of front propagation. The curvature effect, observed at the early stage when the size of the pattern region is small, is crucial for the mechanism of a wavy front. Our explanation of how curvature effect results in a wavy front is shown in Fig. 10(b). When the supercritical number $\varepsilon>0$, the velocity of front propagation increases with the increase in curvature. Assuming that the initial front is a straight line with a protrusion as the disturbance, the protrusion with a curvature $K>0$ has a larger velocity due to the curvature effect, and the two concavities with $K<0$ beside the protrusion have smaller velocities. Therefore, in the following time sequences, the protrusion propagates forward and the curvature increases. This increases the protrusion velocity further and results in a wavy front.

The wavy front is found in the numerical simulation of the model equation. When $\varepsilon=0.02$, the front configuration is circular at the beginning, and then it transforms to a wavy front, as shown in Fig. 10(a). The pattern shows a flower-like shape of the wavy front; meanwhile, the disorder pattern is generated on the wavy front. This gives an explanation of the increase of the velocity of front propagation after the regular pattern transits to an irregular pattern. This is because the flower petal of the wave front has a large curvature which increases the velocity. This theory can also explain why the critical or subcritical front usually forms a regular pattern. Because the subcritical front has a reverse effect on the velocity of front propagation, the protrusion, as shown in Fig. 10(b), has a smaller velocity, which increases the stability of the wave front.

On the one hand, the instability of front propagation makes a wavy front and increases the velocity of front propagation. On the other hand, it induces defect patterns. Figure 11 shows the dynamics of defect generation caused by front instability. Figure 11(a) shows that the initial pattern is arrays of hexagonal cells. We mark the cells at the front by a red line. As the front is propagating, a protrusion is found, which is marked by the red curve shown in Fig. 11(b). Due to the curvature effect, the protrusion propagates faster, while two 


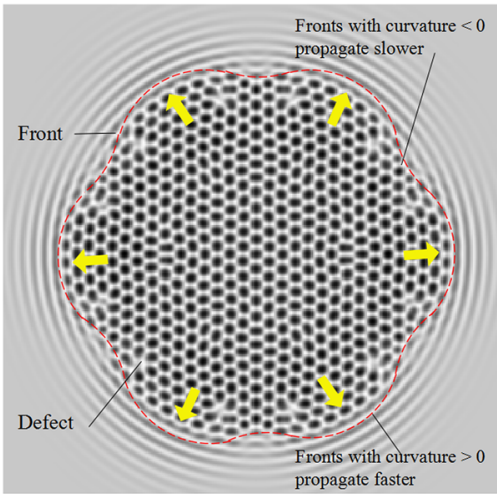

(a)

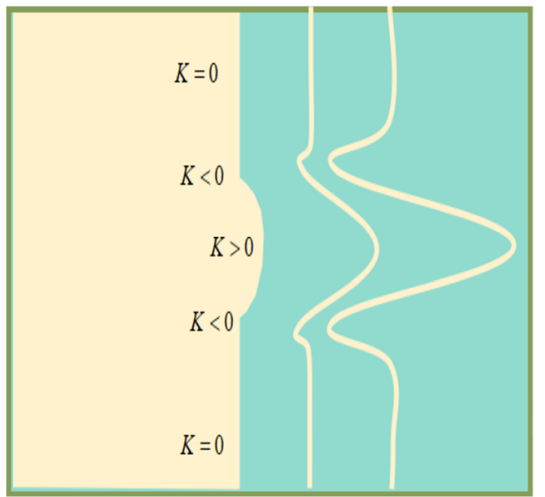

(b)
FIG. 10. The instability of the front: (a) wave front $(\varepsilon=0.02$, $t=110$ ) and (b) mechanism of instability. In Fig. 10(a), the circular front changes to a flow-shaped front and the defect is generated on the front. Figure 10 (b) shows that the front instability is caused by the curvature effect. concavities beside the protrusion propagate slower. In consequence, pattern formation at the concavities is delayed, which breaks the formation order of the pattern. Defects appear at these two concavities, as shown in Fig. 11(c). In Fig. 11(d), we find that the defects consist of two sets of dislocations in different orientations. After the emergence of the disorder, the vertical arrays of cells, as shown in Fig. 11(d), are bent and kept parallel to the front.

Once defects are formed, the newly generated pattern always is in disorder. Figure 12(a) shows the pattern at $t=160$ when many defects can be found after the destabilization of the curve. Figure 12(b) shows the distribution of disturbance amplitude by weighted RMS. It demonstrates that the front is not a smooth curve but with many protrusions. The protrusion has a larger velocity than the concavities around. As the velocity of front propagation is proportional to the front curvature, the maximum velocities are expected to appear at $V_{1}$ and $V_{3}$, as shown in Fig. 12(b). As a result, the newly generated cells surround the concavities, labeled $\mathrm{cl}$ and c2, and form new defects [Fig. 12(c)]. Therefore, we can understand more clearly that the wavy front with nonuniform velocities caused by the curvature effect results in a disorder pattern. In other words, to reduce the disorder, one should reduce the front instability.

\section{Regular pattern generated by a slowly propagating front}

In industrial applications, people may intend to make a regular hexagonal pattern with minimum defects. However, the self-organization of hexagon cells may be accompanied with many defects. The generation of disorder patterns can be induced by the intrinsic effect or extrinsic effect. Therefore, to prepare a regular hexagonal pattern, on the one hand, it is necessary to reduce the intrinsic effect by controlling the temperature difference so that it is close to the critical threshold; on the other hand, it is necessary to restrain the extrinsic effect through some methods, such as using a hexagon vessel or improving the flatness and thermal uniformity of the bottom. Moreover, the temperature difference should be maintained for several hours as the relaxation time. However, disorder cells cannot be avoided because the initial disturbance is not under control during self-organization. The disorder pattern is very stable after its formation.

Through numerical simulation of the model equation, we learn that a single "nucleus" can easily grow into the regular pattern. This process is very similar to the growth of a single crystal, where a single crystal seed is grown into a large single crystal. Similarly, we can generate a regular pattern through the front propagation of regular local initialization. Also, velocity of front propagation should be controlled because we should avoid front instability which may induce disorders. The preparation of the initial local pattern and the control of the velocity of front propagation are accomplished by the cover plates, as shown in Fig. 2.

We propose a new method for generating regular hexagonal Bénard cells. It is implemented through the following steps. First, we raise the temperature difference so that it is close to the critical value until the convective pattern is formed spontaneously. Second,

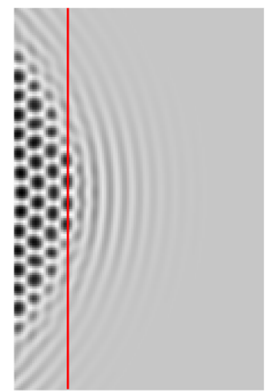

(a)

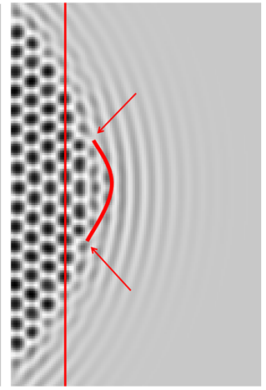

(b)

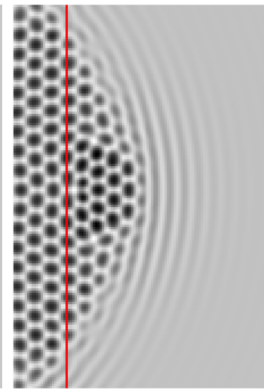

(c)

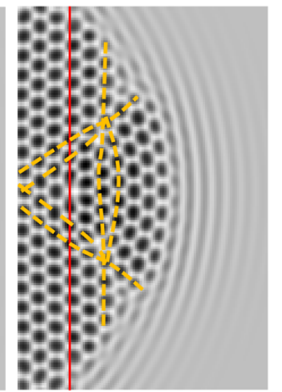

(d)
FIG. 11. The dynamics of front instability and defect generation $(\varepsilon=0.015)$ : (a) pattern at $t=110$, (b) pattern at $t=120$, (c) pattern at $t=130$, and (d) pattern at $t=140$. The protrusion of the front is marked in Fig. $11(\mathrm{~b})$, and the defect is marked in Fig. 11(d). 


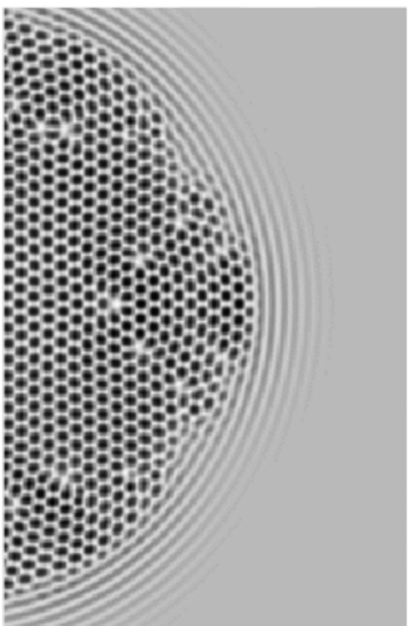

(a)

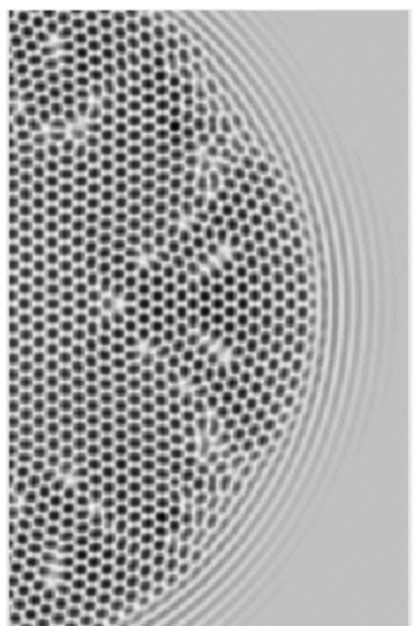

(c)

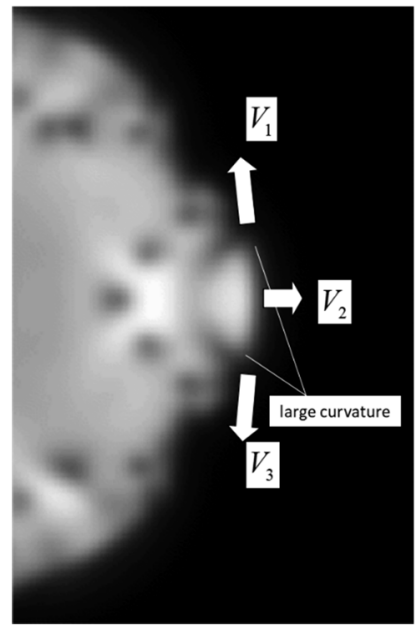

(b)

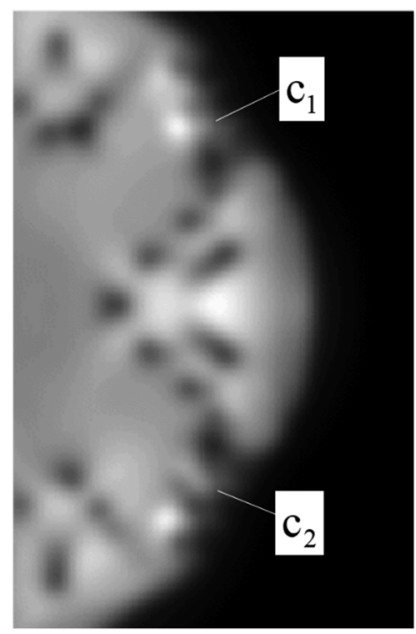

(d)
FIG. 12. The dynamics of front instability and defect generation $(\varepsilon=0.015)$ : (a) and (b) are the pattern and the amplitude of the pattern at $t=160$, respectively. (c) and (d) are the pattern and the amplitude of the pattern at $t=190$, respectively.

we choose the most regular small region as a nucleus and cover the other region by plates, as shown in Fig. 13(a). Therefore, the pattern in the other region vanishes, and only the regular hexagon nucleus survives. Third, we slowly enlarge the convective region by withdrawing the cover plates in the horizontal direction, which makes the front propagate accordingly. Figure 13(b) shows the final pattern in our rectangular vessel. We can see that the amount of defects is greatly reduced.

For a $2 \mathrm{~mm}$ layer of $50 \mathrm{cSt}$ silicone oil, when the temperature difference exceeds the critical value of $4{ }^{\circ} \mathrm{C},{ }^{25}$ the flow will lose stability and naturally form hexagonal cells, with many defects in the naturally formed cells. So we need to choose a regular region as a seed of the hexagon cell. By applying cover plates, the cellular convection under the plates will be effectively suppressed, and only the

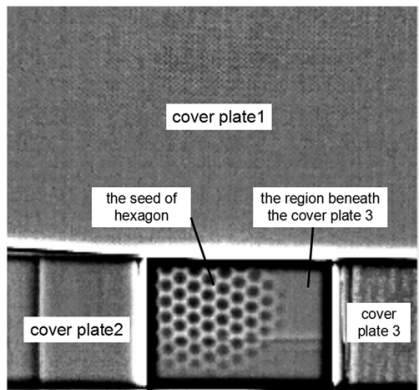

(a)

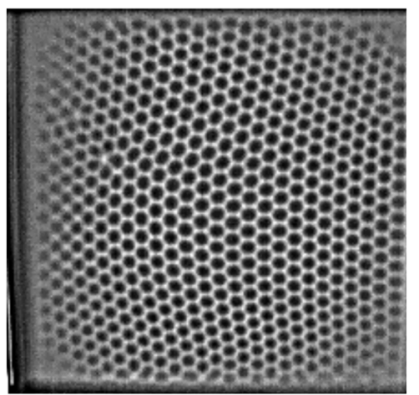

(b)
FIG. 13. Generation of the hexagonal pattern: (a) the seed pattern that is manually initialized by setting the window and (b) the final pattern that is generated by withdrawing the cover plates slowly. The infrared camera is fixed on a tilt to observe the region beneath the cover plate, where the cells are vanished, as seen in Fig. 13(a).

convection near the window is preserved as a seed. This is because the cover plates suppress the convective heat dissipation, which leads to the rise in the interface temperature and the decrease in temperature difference, which is lower than the critical value. When the cover plate is withdrawn slowly, the convective region expands to the whole field. The velocity of front propagation of the hexagon pattern should be controlled very small, which the front instability and the emergence of other disturbance nuclei are restrained, so the newly formed cells are very hexagon patterns.

When withdrawing the cover plate, the smaller the speed is, the more favorable it is to form regular hexagonal cells. The front velocity of hexagonal cells is controlled to a small value by the velocity of the cover plates. On the one hand, it can suppress the instability of front propagation. On the other hand, it can suppress the formation of multiple nuclei in other regions. Theoretically, we need to control the speed of the cover plate so that it is less than the natural speed of the regular pattern's fronts. The nondimensional velocity of natural front propagation is 6.0 near critical, as shown in Fig. 9. The plate velocity is $0.075 \mathrm{~mm} / \mathrm{s}$, as shown in Fig. 13, which demonstrates that the defects will be greatly reduced. This plate velocity corresponds to a front nondimensional velocity of 0.74 , which is $12 \%$ of the natural speed. Therefore, we recommend that the speed should be controlled at $12 \%$ of the natural velocity of front propagation.

\section{CONCLUSIONS}

This paper presents simulations of the evolution of local hexagonal cells to the whole region in Bénard-Marangoni convection using the simplified model equation. We focus on the mechanism of defect generation in order to find a possible method to reduce defects. For comparison, both global random disturbances and the local hexagonal disturbance are simulated. For global random disturbances, the disturbances spontaneously accumulate in scattered multiple nuclei and develop into grain boundaries. For the local hexagonal disturbance, the disturbance is considered as a single nucleus whose front propagates outward until the regular hexagons occupy the whole region. Therefore, a single nucleus can make a regular hexagonal pattern, and multiple nuclei evolve into grain 
boundaries. This proves that the initialization of a single nucleus is helpful in reducing the disorder pattern.

Front propagation and defect formation are also studied by simulations. Defects are generated during the growth of the hexagon region. The propagation of the front is divided into three stages: curvature flow, regular pattern, and irregular pattern. We have found that the curvature of the front accelerates the propagation under the supercritical condition and vice versa under the subcritical condition. Therefore, due to the effect of curvature flow, the protrusion propagates faster than concavities, which forms a wavy front. The wavy front disrupts the order of hexagon formation and causes the irregular pattern at the concavities. It is found that the front of an irregular pattern propagates faster than that of a regular pattern.

Experiments demonstrate that there exist the local hexagonal pattern and the front propagation in self-organized BénardMarangoni convection. We have researched propagation velocity in different pattern orientations and under different supercritical numbers. The results show that the velocity increases with the increase in the supercritical number and the parallel pattern propagates faster than the vertical pattern. The velocity of the front is accelerated after the emergence of the disorder pattern, which is qualitatively consistent with the simulation. Inspired by the simulation, we have developed a method for generating a regular hexagon pattern. It is achieved by exerting local hexagonal initialization and controlling the velocity of front propagation to suppress front instability. Experimental results show that this method greatly reduces the amount of defects. It may be also useful for creating a regular pattern in other nonequilibrium systems.

\section{ACKNOWLEDGMENTS}

This work was supported by the National Natural Science Foundation of China (Grant No. 11502271), the Joint Funds of the National Natural Science Foundation of China (Grant No. U1738116), the Strategic Priority Research Program on Space Science, the Chinese Academy of Sciences: SJ-10 Recoverable Scientific Experiment Satellite (Grant Nos. XDA04020405 and XDA04020202-05), and the China Manned Space Engineering Program.

\section{REFERENCES}

${ }^{1}$ H. Bénard, "Les tourbillons cellulaires dans une nappe liquide," Rev. Gen. Sci. Pures Appl. 11, 1261-1271 (1900).

${ }^{2}$ Lord Rayleigh, "On convection currents in a horizontal layer of fluid, when the higher temperature is on the under side," London, Edinburgh, Dublin Philos. Mag. J. Sci. 32(192), 529-546 (1916).

${ }^{3}$ J. R. A. Pearson, "On convection cells induced by surface tension," J. Fluid Mech. 4(5), 489-500 (1958).
${ }^{4}$ A. Cloot and G. Lebon, "A nonlinear stability analysis of the Bénard-Marangoni problem,” J. Fluid Mech. 145, 447-469 (1984).

${ }^{5}$ P. Colinet, J. C. Legros, and M. G. Velarde, Nonlinear Dynamics of SurfaceTension-Driven Instabilities (Wiley-VCH, 2001).

${ }^{6}$ J. Swift and P. C. Hohenberg, "Hydrodynamic fluctuations at the convective instability," Phys. Rev. A 15(1), 319 (1977).

${ }^{7}$ M. Bestehorn, "Phase and amplitude instabilities for Bénard-Marangoni convection in fluid layers with large aspect ratio," Phys. Rev. E 48(5), 3622 (1993).

${ }^{8}$ D. Fan, X. Xia, H. Ma, B. Du, and Q. Wei, "Honeycomb-patterned fluorescent films fabricated by self-assembly of surfactant-assisted porphyrin/polymer composites," J. Colloid Interface Sci. 402, 146-150 (2013).

${ }^{9}$ D. W. Janes, J. M. Katzenstein, K. Shanmuganathan, and C. J. Ellison, “Directing convection to pattern thin polymer films," J. Polym. Sci., Part B: Polym. Phys. 51(7), 535-545 (2013).

${ }^{10}$ I. Nejati, M. Dietzel, and S. Hardt, "Exploiting cellular convection in a thick liquid layer to pattern a thin polymer film," Appl. Phys. Lett. 108(5), 051604 (2016).

${ }^{11}$ E. L. Koschmieder and D. W. Switzer, "The wavenumbers of supercritical surface-tension-driven Bénard convection,” J. Fluid Mech. 240, 533-548 (1992).

${ }^{12}$ A. M. Kvarving, T. Bjøntegaard, and E. M. Rønquist, “On pattern selection in three-dimensional Bénard-Marangoni flows,” Commun. Comput. Phys. 11(3), 893-924 (2012).

${ }^{13}$ P. Cerisier, S. Rahal, and B. Billia, "Extrinsic effects on the disorder dynamics of Bénard-Marangoni patterns,” Phys. Rev. E 54(4), 3508 (1996).

${ }^{14}$ P. Cerisier, S. Rahal, and N. Rivier, "Topological correlations in BénardMarangoni convective structures," Phys. Rev. E 54(5), 5086 (1996).

${ }^{15}$ M. F. Schatz and G. P. Neitzel, "Experiments on thermocapillary instabilities," Annu. Rev. Fluid Mech. 33(1), 93-127 (2001).

${ }^{16}$ S. W. Morris, E. Bodenschatz, D. S. Cannell, and G. Ahlers, "Spiral defect chaos in large aspect ratio Rayleigh-Bénard convection," Phys. Rev. Lett. 71(13), 2026-2029 (1993).

${ }^{17}$ A. K. Tripathi and D. Kumar, "Stripe patterns: Role of initial state and boundary conditions,” Phys. Rev. E 90(2), 022915 (2014).

${ }^{18}$ M. F. Schatz, S. J. VanHook, W. D. McCormick, J. B. Swift, and H. L. Swinney, “Onset of surface-tension-driven Bénard convection," Phys. Rev. Lett. 75(10), 1938 (1995).

${ }^{19}$ W. V. Saarloos, "Front propagation into unstable states," Phys. Rep. 386(2), 29-222 (2003).

${ }^{20}$ B. C. Ponedel, H. C. Kao, and E. Knobloch, "Front propagation in weakly subcritical pattern-forming systems," Phys. Rev. E 96(3), 032208 (2017).

${ }^{21}$ A. A. Nepomnyashchy, "Nonlinear dynamics of fronts," in Pattern Formation at Interfaces (Springer, Vienna, 2010), pp. 57-103.

${ }^{22}$ A. Tzella and J. Vanneste, "Front propagation in cellular flows for fast reaction and small diffusivity," Phys. Rev. E 90(1), 011001 (2014).

${ }^{23}$ C. Storm, W. Spruijt, U. Ebert, and W. van Saarloos, "Universal algebraic relaxation of velocity and phase in pulled fronts generating periodic or chaotic states," Phys. Rev. E 61(6), R6063 (2000).

${ }^{24}$ J. Fineberg and V. Steinberg, "Vortex-front propagation in Rayleigh-Bénard convection,” Phys. Rev. Lett. 58(13), 1332 (1987).

${ }^{25} \mathrm{D}$. Wu, L. Duan, and Q. Kang, "Wavenumber selection by Bénard-Marangoni convection at high supercritical number," Chin. Phys. Lett. 34(5), 054702 (2017). 